\title{
RELATION BETWEEN STRUCTURAL AND BEHAVIOR PSYCHOLOGY
}

\author{
BY A. P. WEISS \\ Ohio State University
}

The purpose of this paper is to show that the problems in the study of mind which are ordinarily the problems of the structural psychologist, may be studied from the behavioristic point of view, in accordance with the methods employed in the natural sciences and with a greater degree of simplicity than is possible from the structuralistic point of view. No attempt will be made to determine whether behaviorism is or is not psychology. The title of this paper might also have been 'The Value of the Objective as Compared with the Subjective Method in Psychology' were it not for the fact that the terms 'objective' and 'subjective' because of their uncertain connotation, are in evil repute both in psychology and in all the social sciences.

\section{Problem of Structural Psychology}

To state the structuralists' problem is not an easy one if justice is to be done to all the psychologists who are representative of this school. There are, however, certain fundamental conceptions which are characteristic of structuralism that are generally accepted, and it is to these that the discussion will be limited.

By the term structural psychology is meant the type of investigation which assumes that there is an existential datum called mind or consciousness, within the totality of which ultimate differences may be discriminated which are given the names of (I) sensations, (2) images, (3) affections. These are usually regarded as the elements into which consciousness may be analyzed. The aim of the structural psychologist who accepts this analysis is to describe consciousness, in all its 
complexity, in terms of these three fundamental classes of consciousness. There is, of course, considerable difference of opinion between structuralists as to just how these classes are to be defined, and indeed, whether there are no more and no less than three elementary categories. But for our purpose these details may be disregarded. It is also irrelevant for us whether it is held that consciousness is present in a situation in which sensations, images and affections, as such, are absent. It is sufficient if we recognize clearly that the structuralist aims to describe the structure of the mind or consciousness, under the manifold conditions of present-day life, in terms of whatever elements he may have set up, and that he considers the descriptive phase of his investigation as complete when he has done this. The explanatory phase of the structuralist problem is the determination of the neural correlates of the conscious states which his analysis reveals.

The direct method by which the structuralist analyzes a conscious complex into its elements is that of introspection. This cannot be done satisfactorily under normal conditions. It is therefore customary to create an experimental situation similar to the one to be analyzed, in which the observer is asked to report in language the mental states that were present during the experiment. These reports of the mental states are then treated statistically. The experimenter assumes that the verbal reports of mental states are not the mental states which the observer introspected, but that the reports are merely expressions which describe the character of these mental states.

There is considerable difference of opinion among structuralists themselves as to whether introspection gives equally valid results in all cases where it may be used. Some maintain that even the most complex mental states may be analyzed by the introspective method, while others maintain that introspection modifies complex conscious experiences so that the elements which it reveals may not be present in the actual experience which is being investigated. 


\section{Origin and Problem of Behavior Psychology}

As the applications of structural psychology became more numerous in the various applied fields of psychology such as education and medicine, the question as to the relation between conscious states and action arose. An increasing number of psychologists believed that sensations, images, memories, thought, emotion, affection, etc., were the causes or invariable antecedents of certain forms of human behavior. This naturally led to the question as to how consciousness was related to changes in the form of human behavior. Now, since no one denied that, in order to modify behavior, a corresponding change in the neural correlates of consciousness must take place, this inquiry necessarily came to be stated as follows: How can consciousness change the direction of a nervous process? In its most general form this question is an inquiry into the relation between mind and body. In animal psychology the mind-body problem is not an acute one for the zoölogist-psychologist who studies animal behavior; and the success which these investigators have had in explaining some of the most complex forms of animal behavior in purely neural terms (tropisms, reflexes, instincts) has led some of the psychologists to ask whether human behavior could not be placed in the same category with animal behavior and explained without the introduction of a mental or conscious factor.

The attempt to answer this question by the application of the behaviorist point of view to such relatively simple forms of human behavior as reflexes and automatic action was crowned with considerable success, but for the more complex behavior of learning the strictly neural explanation is not readily accepted by psychologists. It will not be necessary at this stage to state the behaviorist problem in detail except to indicate that the behaviorist is concerned with determining the properties and laws of the neuro-muscular system, of which the introspective reaction is a part.

Briefly, we may state the problem of the structuralist as an attempt to answer the question: What are our mental states 
and how do they come about? while the behaviorist problem is: What are our actions and how do they come about?

The following paragraphs are devoted to a discussion, from the behaviorist point of view, of those factors of structural psychology which give it its distinctive character: namely, the relation between mind and body or the character of consciousness; and the character of introspection.

\section{Behayiorism and the Mind-body Problem}

Discussion by psychologists of the mind-body problem has led to the formulation of the three following positions:

I. Consciousness does not enter as a causal agent into such actions as reflexes and instincts, but it does function in what is usually known as intelligent or voluntary action.

2. Consciousness cannot be regarded as the invariable antecedent to any kind of action whatsoever.

The first position is the one usually taken by popular psychology and the group of psychologists known as "functionalists," while the second position is the one maintained by behaviorists. The position of the structuralists is usually known as the double-aspect view, in which there is,

3. Parallelism between conscious processes and neural processes without a causal relationship between them.

This position is best described by the following quotation:

"Our own position has been that mind and body . . . are simply two aspects of the same world of experience. They can not influence each other because they are not separate or independent things. For the same reason, however, whenever the two aspects appear, any change which occurs in the one, will be accompanied by a corresponding change in the other."1

The third view may be regarded as an intermediate position between 1 and 2 . That is to say a functional relationship does exist between mind and body but only in the mathematical sense of the term function, as when we speak of the volume of a sphere as a function of its radius.

If this functional relationship between consciousness and behavior is a simple one it would be useful for the behaviorist, because consciousness could then be used as a measure of

I Titchener, E. B., 'A Text-Book of Psychology;' I912, p. 9. 
behavior, no matter what the ultimate connection between mind and body might be.

Setting aside all speculation about this connection we find that the facts in the case may be stated in the following propositions:

I. Consciousness (the totality of our sensations, images and affections) is a purely personal experience and has no scientific value or validity unless it is expressed in some form of behavior, such as speech or other form of representation.

2. Many forms of behavior (reflexes, automatic action) are not accompanied by consciousness which can be unambiguously analyzed.

3. The consciousness which does accompany a given form of behavior varies from one observer to another, and at different times for the same observer.

4. Complex mental processes, such as reasoning and invention, are more than the mental states into which they may be analyzed. They have a social reference which no amount of introspection will reveal. This social reference is measured by the comparison of the individual's behavior, either with his ordinary behavior or with that of other individuals.

From the above propositions the behaviorist feels justified in making certain deductions:

From proposition I: If consciousness must be expressed in some form of behavior before it becomes a scientific datum, then consciousness is in the last analysis a classification and a study of behavior.

From proposition 2: If there are many forms of behavior (reflexes, automatic action) which are important from a social or individual standpoint, which are not accompanied by consciousness, then consciousness is not a function (mathematical) of all forms of behavior.

From proposition 3: If the consciousness which does accompany a given form of behavior, varies from one person to another, or for the same person at different times, then consciousness is either an independent variable of the behavior, or a dependent variable of highly complex formula. In neither case is it any better measure of behavior than the 
behavior itself, and consciousness then becomes superfiuous as a means for predicting behavior.

From proposition 4: If complex mental processes such as reasoning or invention are more than the mental states into which they may be analyzed, then in this case too the analysis of consciousness does not make it possible to predict the individual's behavior.

In view of the above implications, the very simplest assumptions as to the quantitative relations.between sensations, images and affections which characterize an individual's conscious complexes, it is impossible to predict what his mental states will be after an hour. An individual's behavior, however, can be predicted with much greater certainty than can the character of the conscious processes which go with it. A daily photographic record, for example, will show greater uniformities in the behavior of a given individual so far as the socially significant factors are concerned, than will the daily introspective descriptions of the consciousness which accompanies his behavior.

It would seem then that the sensations, images and affections which one experiences are more variable than is behavior, since by the term behavior we usually refer to only the grosser bodily movements and to the speech reactions. The finer changes in muscle contractions, as in respiration, digestion, vascular and glandular changes, are ordinarily not considered as socially significant.

For the behaviorist, then, the double-aspect view of the structuralist is of no value, since the function (consciousness) of the behavior is more variable than the behavior itself.

\section{Consciousness an Inference Derived from the Intro- SPECTIVE REACTION}

The structuralist is usually disposed to consider the investigations of the behaviorist as quite outside of his field, because consciousness or mind is something entirely distinct from the contraction of a system of muscles. In the last analysis, however, the consciousness of his observer which the structuralist regards as the unique phase of his investi- 
gation, is only an inference from the verbo-motor behavior which is called introspection. Structuralists' experiments are controlled reactions to stimuli just as in any behavior experiment. The sensations, images and feeling that are described, are not experienced by the structuralist. They are inferred from the behavior of his observers.

When for instance, an introspective reaction reads, " $\mathrm{My}$ attention fell upon the central projection, and vocal-motor imagery of 'Gosh, that's ugly' occurred. I was aware of contraction of my brows and unpleasantness." The structuralist infers that these speech reactions are descriptions of the consciousness that he has when he makes the same speech reactions. To every introspective report of his observer, the structuralist adds, at least implicitly, the speech reaction, namely: "The introspective report of my observer completely describes certain conscious processes of which I am myself conscious or aware, when I make the same speech reactions."

In other words, psychologists have agreed among themselves that an introspective report is more than a reaction; it is a verbal reaction plus some kind of a conscious process, which may be either sensorial, imaginal or affective. The behaviorist may well ask of what scientific value is this habit of supplementing, in a methodological sense, the observer's reaction by another reaction of an entirely different type (consciousness) which the observer did not report. By inferring a conscious correlate to the introspective report-the experimenter is not able to derive anything more from the report than is expressed in it. The manual and introspective reactions of the observer in a psychological experiment can be classified just as minutely and scientifically without inferring a conscious correlate, as they can with it.

\section{BEHAVIORISM AND INTROSPECTION}

From the behavioristic standpoint, introspection may be regarded as an example of habit formation or learning. An untrained observer cannot make these introspective speech reactions in a manner which is constant and uniform enough to permit of statistically treating the results. In order that 
these supplementary speech reactions may become uniform enough to admit of statistical analysis, two methods of simplifying the results are usually employed:

I. The observer passes through a preliminary period of training in which the reaction time of relevant speech reactions (report of imagery, kinesthesia, etc.) is reduced to the experimental requirements, and those speech reactions which are irrelevant are given an opportunity to disappear. The relevancy of a given speech reaction is usually determined beforehand according to the specific aim of the experiment.

2. The experimental situation is so modified by supplementary situations in the form of instructions, questions or other controls that the required uniformity of the introspective reactions is secured.

The first method is the one with which we will especially concern ourselves in the following discussion.

As already indicated, the total reaction of an organism to even the simplest situation is very complex. Respiration, secretion, circulatory effects, incipient and minute bodily and visceral movements, are always added to the particular reaction (the discrimination of two points, for instance) which might be called the major reaction of the experiment. The minor reactions by themselves would escape notice, but the speech mechanism is a rather sensitive index as to the character of these minor reactions. Permitting a free verbal report after each trial or series of trials in an experiment, reveals more as to the character of the total reaction than can be learned from the major reaction alone.

Organic and kinesthetic reactions which would escape observation entirely are thus easily revealed by the speech reaction. If, for instance, an observer who has been trained according to method ( 1 ) reports a 'kinesthetic sensation of movement of the neck' that report from the behaviorist point of view would mean, 'an incipient or slight movement of the head,' provided his methods of measuring such movements were accurate enough. In this way the verbal reaction 'kinesthetic sensation of neck movement' really indicates that the receptors in this case are located in the neck muscles. 
The speech reaction may therefore be regarded as a function of particular kinesthetic and organic reactions which ordinarily would escape observation.

To report kinesthetic and organic conditions through speech is really a highly specialized example of habit formation. Indeed introspection, whatever its kind, from this point of view may be regarded as a continual process of training in the formation of habits. For this reason many years of training are necessary even for those who have special aptitude in this direction, and many persons never learn to introspect well.

The training of an individual in introspection is, therefore, a process of:

I. Training in discrimination so that weak stimuli in obscure receptors lead to verbal reactions.

2. Substituting for the usual anatomical and physiological descriptions (head, neck, arms, muscles, viscera, etc.) the special terminology of the structuralist (sensations, images and affections).

The relation of these obscure receptors to the major reaction of the experiment may be of a highly adventitious character. A trained observer does not react exclusively to the experimental situation-one is almost tempted to say that he reacts to everything but the experimental situation. An experiment with trained observers measures principally the training of the observers, it does not necessarily reveal what the normal individual could be expected to do under similar situations or conditions.

From the behavioristic standpoint, then, introspection may be regarded as a greatly augmented reaction to a given situation. The observer not only reacts to the situation, for example, by pressing a key, but he is also asked to react by speech to the stimulation of many secondary and obscure receptors, to which, under normal conditions, no such reaction is made. 


\section{Heuristic Value of Introspection}

When the structuralist experiment is stripped of its conscious reference and regarded from the standpoint of objective science, its twofold character immediately comes to light as may be seen from the following quotation which is part of the conclusion of a long and painstaking series of experiments "On the Analysis of a Phase of the Process of Classifying."

"The essence of the process of classifying, as this process occurred in our experiments, consisted in the manner of our observer's perceiving the object which he had been instructed to classify. This manner of perceiving consisted in the fact that the region of essential group features-were stressed in consciousness, and these regions behaved in consciousness in a fashion which depended upon their resemblance or lack of resemblance to the corresponding features in the group members. In the former event, the regions in question passed in and out of consciousness in rapid and ready fashion, without retarding the course of attention. In the latter case, on the other hand, the course of attention was arrested sharply; these regions often persisted in consciousness, and they were frequently accompanied sooner or later by more or less focal and intensive kinesthetic, organic and affective contents which functioned in their conscious settings as definite rejectings of the figure."

Will the above conclusions, we may ask, enable a botanist or zoologist to classify his specimens more effectively, or will the scientist know any more about classifying than he did before he read the article? The question may even be asked, whether this particular experiment is really an investigation of the process of classifying. From the behavior point of view at least, the investigation would be regarded as an experiment in discrimination and habit formation, carried out under the following conditions:

An experimental situation was created which approached the conditions under which people make classifications, except that the classifications are rather more than ordinarily difficult. A number of observers who had been carefully trained to respond verbally to the weak stimulation of obscure receptors, were asked to perform a double task; to classify the cards, and also to react to the obscure receptors by verbal

1 Fisher, Sara C., 'An Analysis of a Phase of the Process of Classifying,' Am. J. of Psychol., 1917. 28, p. 115.

I have chosen this particular illustration because I regard it as one of the best structuralist experiments that has appeared for some time and I need hardly say that no criticism is intended either of it or the method by which the investigator carried it to completion. 
reactions which conformed to a more or less uniform terminology. An analysis of the author's conclusion quoted above, in behaviorist terms, may be stated in the following propositions:

I. The observers were able to classify the cards.

2. The observers reacted either to the similar or to the dissimilar features of the cards. The reactions to the similar features were relatively simple; the reactions to the dissimilar features were supplemented by verbal reactions to obscure kinesthetic and organic receptors.

The whole emphasis of the experiment is placed upon what the behaviorist would call the reactions to obscure receptors. The actual process of classifying the cards was merely a device to obtain some degree of uniformity in the verbal reactions to these obscure receptors. From these considerations we can see why the experiment has so little significance for the scientist who really wishes to increase his classifying efficiency. The development of the ability to react to the weak stimulation of obscure receptors may be of value in the above experiment as a means of determining when the investigation of the classifying process was being supplanted by the minor reactions which have no direct bearing on the process of classifying.

That is to say, the presence of sensations, images and affections, indicate that there is something wrong with the experiment.

In ordinary scientific observation, the aim is to eliminate the unessential or obscure so that the effect of the major condition can be observed in isolation. Under behavioristic methods, however, if it is desired to investigate the minor reactions independently, then every effort is made to isolate them and they of course then become the major reactions. But the introspective reactions in this particular experiment would be regarded by the behaviorist as a disturbing factor.

In our effective adjustments to our environment, we do not have sensations, images or affections. When we stop to introspect as to the character of our consciousness in a given situation, our reactions to that situation become to this 
extent irrelevant. The writer has frequent occasions to design modifications in apparatus used by the students working on original problems. Having also some epistemological interest, it often happens that he begins to introspect on the method by which the designing process is going on. When this happens, he might as well stop. Introspection seriously interferes with the designing activity. The reactions, incipient though they be, which are called introspections, change the character of the designing activity to such an extent that it can no longer be called the designing activity. This is gone; he is merely reacting to a new situation by making aimless sketches and many incipient verbomotor processes which if recorded might read, "Bronze contact, visual image of tuning fork, kinesthetic sensations of neck muscles, slight unpleasantness, image of $\mathrm{Mr}$. M. before chronoscope, visual image of Prof. X. rushing upstairs, etc."

None of these reactions would have occurred had he actually completed the original design. This is, of course, not a unique situation. Under the ordinary conditions, sensations, images and affections are absent. The average man probably never has them; he must be trained to have them in the same way that we must be trained to read and write. Introspection is only one of the ways by which we may react to a situation. How the reactions themselves originate, or what the conditions are under which they become modified, is not revealed by introspection.

\section{Tautological Character of Introspection}

By selecting a somewhat simpler activity than that of "classifying" it can be shown that the introspective records are merely special terms for particular classes of reactions. Suppose we take the process of recognition from the structuralist standpoint. We may assume that the recognitive consciousness is made up of a characteristic pattern of sensations, images and affections. Suppose the experiment is one in which we are to select a card $(A)$ which has been previously examined, from a series of similar cards which have not been previously examined. We wish to know whether 
the card $(A)$ has been recognized. To do this, the behaviorist would inspect the introspective reactions of the observer. Suppose the verbal reactions to the card $(A)$ to be the following. "I have seen it before; I have a visual image of it as it appeared when I saw it for the first time; I have the feeling of familiarity; I have organic and kinesthetic sensations (images?), which mean that I am handling it in a more energetic and discriminative fashion than the other cards; etc." To the new cards the verbal reactions might be as follows: "I have never seen these cards before; the kinesthetic and organic sensations are different than for card $(A)$; they do not carry the meaning of familiarity; the visual and auditory imagery is different than for card $(A)$; etc." It is not supposed that this is the complete introspective record, and we disregard for the present such objective measures as the reaction time, or of the bodily movements which might have been made.

If we regard the introspections from the purely objective side as being muscle contractions of the speech mechanism which produce the various sounds which we call words or speech, we can immediately see that the observer has reacted differently to the card $(A)$ than to the other cards. It is not necessary to interpolate a conscious process (of recognition) in order to see this. The fact is, that if the observer has used the identical words to describe both the $(A)$ and the new cards, we should be obliged to conclude that there was no difference in the observer's consciousness of the $(A)$ and the other cards. That is to say, a difference in consciousness can only be inferred from a difference in behavior. Moreover, the interpolation of consciousness does not explain how it came about that the card $(A)$ was reacted to in a manner different from that of the other cards. The introspective record merely reiterates the fact that the observer did react differently. This could have been seen directly by regarding the report of the introspective reactions as what, in fact, they are-objective reactions of the speech type. Because the observer reacted differently to the $(A)$ than to the other cards, the experimenter says he has given a recognizing 
reaction. We cannot say he reacted differently because he recognized, but that he recognized because he reacted differently. In this sense the inference of the conscious process of recognition is tautological.

\section{Structuralism a Phase of Behaviorism}

The structuralist point of view can, of course, be consistently maintained. There is a justification for inferring the existence of a conscious correlate for at least some of our actions, but the heuristic value of this assumption seems doubtful when it is shown that behaviorism is not less discriminative or descriptive than structural psychology, and in addition it has the promise at least of a system whose parts are causally related to each other in the sense that a temporal description of a given form of behavior is more uniform than the temporal description of the conscious states which are said to go with it. If this is true then the law of Parsimony will operate to eliminate the unnecessary assumption of a conscious correlate for behavior (implied by introspection). When we recall how we are being exhorted to recognize the unconscious, subconscious, higher thought processes, unanschaulichen Bewusstseinsinhalt, one wonders whether this process of elimination has not gone further than we suspect.

For the behaviorist the structuralist's classifications do not lead to a solution of the problem, What are our actions and how do they come about? or How do the manifold social adjustments of the adult grow out of the relatively simple reactions of the child? Even if the objective side of the structuralist problem be further developed, the neural correlate of conscious processes will only be a very special problem in behavior. Sensations, images, affections, emotions, will, the self, recognition, etc., so far as science is concerned are only special instances of receptor-effector activity. While this is of course very generally recognized by psychologists, the further assumption that these forms of receptor-effector activity are highly significant for our understanding how man is able to make his effective adjustment to his environment, is not warranted, because the analysis and isolation of such a 
neural correlate does not indicate how the neural correlate itself came to have the configuration that it has.

This, of course, can only be done by a genetic study of the particular type of reaction. For example, when the structuralist has determined the neural correlate for the process called "recognition," his explanation is complete. For the behaviorist this is merely a type of reaction whose effectorreceptor phase is described by the proposition: To objects to which we have reacted once, we do not react in identically the same way the next time they are presented, even though the stimulus conditions approach identity for both occasions. This is merely a statement of fact which becomes valuable only when stated quantitatively.

The more important scientific question: How does this action come about, can only be answered by increasing our knowledge of neural function.

It is in the above sense that we may regard the introspective reaction of the structuralist as only a part of the total problem of determining how man makes his manifold adjustments to his environment.

\section{Basis OF BEHAVIORISM}

In order to state the position of the behaviorist more concretely, the following assumptions as to the explanation of human behavior are presented.

I. The resistance of neurons varies with function.

2. Every receptor is directly connected by a neural chain with a restricted effector system, and through varying degrees of indirectness to many other effector systems.

3. One nervous process will modify the character of other nervous processes that occur together with it.

4. Certain configurations of neural connections between receptors and effectors are inherited, some are acquired.

These assumptions are not essentially different than those formulated by Max F. Meyer. ${ }^{1}$

From these four laws or assumptions it is evident that there is a possibility of greater variations in the response of an

' 'The Fundamental Laws of Human Behavior,' 19ri. 
organism than is usually included under what the structuralists call the study of mind.

Each of the four propositions represents a series of problems which may be experimentally handled in the same manner that an experiment in any other science would be conducted. There need be no quarreling about consciousness or as to whether there is or is not such a thing, since it will make no difference in the effectiveness of the work of the behaviorist or any scientist how the question may finally be settled. In their present form the propositions represent little more than a program for future work. Each proposition will be divided and subdivided and coefficients will be supplied to the various derived propositions. How fruitful the analysis will be cannot of course be predicted, but if structural psychology, with all the painstaking and careful work that has been done on sensorial and imaginal processes, has not yet developed a definition of sensation upon which two psychologists will agree, then behaviorism can expect a considerable degree of tolerant good will.

\section{SUMMARY}

I. The close academic relationship between structural psychology and behaviorism is due to the popular belief that mind and body are related in such a way that mind produces or modifies behavior.

2. A conscious state which is not expressed in some form of behavior is, so far as science is concerned, non-existent. The inference of consciousness is, therefore, unnecessary, since in the last analysis behavior is the only thing that can be classified.

3. For the behaviorist the introspective reaction is only the habit of being able to react by speech, more or less adventitiously, to the weak stimulation of obscure receptors.

4. Even if it is admitted that the special introspective habits are correlated with conscious processes, the latter are so variable that they cannot be used as an index from which to predict behavior which is socially or scientifically significant.

5. The method of introspection favors the reactions to obscure stimuli and in a corresponding degree decreases the 
reliability of the major reaction for the investigation of which the experiment is designed.

6. Behaviorism presents as manifold possibilities of analysis and classification as does structuralism, and has the added advantage that its phenomena can be represented as a causal series in the same sense as we now speak of causal relationship in the natural sciences.

7. When the behaviorist solves the problem: How do our actions come about? all the problems of the structuralist will also be solved.

8. The relation between structuralism and behaviorism is such that they may exist side by side. This will occur only when the fundamental conceptions underlying both methods are not very closely scrutinized or where the individual is not particularly interested in the theoretical implications of either the one or the other method. 